\title{
Analyzing the Effects of Particle Density, Size and Size Distribution for Minimum Fluidization Velocity with Eulerian-Lagrangian CFD Simulation
}

\author{
Janitha C. Bandara $^{1} \quad$ Marianne S. Eikeland $^{1} \quad$ Britt M. E. Moldestad $^{1}$ \\ ${ }^{1}$ Faculty of Technology, Natural Sciences and Maritime Sciences, University College of Southeast Norway \\ \{Janitha.bandara, Marianne.Eikeland, britt.moldestad\}@usn.no
}

\begin{abstract}
Fluidized bed reactor systems are widely used due to excellent heat and mass transfer characteristics followed by uniform temperature distribution throughout the reactor volume. The importance of fluidized beds is further demonstrated in high exothermic reactions such as combustion and gasification where fluidization avoids the hot spot and cold spot generation. A bed material, such as sand or catalyst, is normally involved in fluidized bed combustion and gasification of biomass. Therefore, it is vital to analyze the hydrodynamics of bed material, especially the minimum fluidization velocity, as it governs the fluid flowrate into the reactor system. There are limitations in experimental investigations of fluidized beds such as observing the bed interior hydrodynamics, where CFD simulations has become a meaningful way with the high computer power. However, due to the large differences in scales from the particle to the reactor geometry, complex interface momentum transfer and particle collisions, CFD modeling and simulation of particle systems are rather difficult. Multiphase particle-in-cell method is an efficient version of Eulerian-Lagrangian modeling and Barracuda VR commercial package was used in this work to analyze the minimum fluidization velocity of particles depending on size, density and size distribution.
\end{abstract}

Wen-YU-Ergun drag model was used to model the interface momentum transfer where default equations and constants were used for other models. The effect of the particle size was analyzed using monodispersed Silica particles with diameters from 400 to 800 microns. Minimum fluidization velocity was increased with particle diameter, where it was $0.225 \mathrm{~m} / \mathrm{s}$ for the 600 microns particles. The density effect was analyzed for 600 microns particles with seven different density values and the minimum fluidization velocity again showed proportionality to the density. The effect of the particle size distribution was analyzed using Silica. Particles with different diameters were mixed together according to pre-determined proportions as the final mixture gives a mean diameter of 600 microns. The 600 microns monodispersed particle bed showed the highest minimum fluidization velocity. However, some particle mixtures were composed with larger particles up to 1000 micron, but with a fraction of smaller particles down to
200 microns at the same time. This shows the effect of strong drag from early fluidizing smaller particles. The only variability for pressure drop during packed bed is the particle size and it was clearly observed in all three cases.

Keywords: $\quad$ Fluidization, Bioenergy, Particle properties, Minimum fluidization velocity

\section{Introduction}

Fluidization occurs whenever a collection of particles is subjected to an upward fluid flow at a sufficient flowrate where the gravity and inter-particle forces are in counterbalance with the fluid drag force (Horio 2013). The fluidized bed technology was first introduced in the petroleum industry for catalytic cracking processes, which later penetrated into energy, environmental and processing industry (Horio 2013, Winter and Schratzer 2013, Vollmari, Jasevičius et al. 2016). The technology enhances the gas-solid contact and mixing, which leads to increased heat and mass transfer characteristics. Further, it guarantees the homogeneous temperature and concentrations throughout the reactor, which increases the possibility and reliability of scaled up operation. Good control over solid particles, large thermal inertia of solids (Esmaili and Mahinpey 2011), increased efficiency, reduced emissions and wide range of operating conditions are additional advantages of the fluidized bed systems (Winter and Schratzer 2013). The importance of the fluidized bed technology is highlighted specially in exothermic reactions such as biomass combustion as it avoids hot spot and cold spot generation due to intense mixing and particle collision. Hot spots lead to ash melting followed by agglomeration and clinkering (Behjat, Shahhosseini et al. 2008, Horio 2013) whereas cold spots reduces tar cracking and thus, reduced gas quality.

Bio-energy is the fourth largest energy source, which accounts for $10 \%$ to $14 \%$ of the world energy profile (REN21 2016). The lignocellulosic fraction of the biomass is the major contributor of bioenergy. In contrast to the simple, inefficient and small-scaled combustion practices, there is a tendency to use advanced technologies such as fluidized bed gasification followed by either heat \& power generation or liquid fuel synthesis. However, due to low density, large particle size and extreme shapes of the particles, 
biomass is difficult to fluidize alone (Cui and Grace 2007). Therefore, biomass fluidized bed combustors and gasifiers are operated with the assistance of fluidizing materials such as sand, alumina, catalysts etc., which is known as bed material (Fotovat, Ansart et al. 2015). Hence, it is meaningful to study the fluidization behavior of bed materials as it principally governs the bed hydrodynamics. Bubbling fluidization stands slightly above the minimum fluidization. Hence, it is important to manipulate the minimum fluidization velocity in bubbling fluidized bed gasification systems, because it governs the mass flowrate of gasifying agent into the reactor system.

The fluidization properties are governed by both particle properties such as particle size, particle density, particle shape etc. and fluid properties (Fotovat, Ansart et al. 2015). However, there can be additional effects from the bed diameter, geometry, aspect ratio and distributor design as well. The transition superficial gas velocity from fixed bed to fluidized bed is referred to as the minimum fluidization velocity, which is one of the most important parameters in the design of fluidized beds (Coltters and Rivas 2004). Depending on Geldart's powder classification and superficial gas velocity, particles tend to fluidize in homogeneous, bubbling, slugging or sprouting beds (Geldart 1973).

Computational Fluid Dynamics (CFD) simulations are beginning to appear in a meaningful way with the tremendous growth in computer power along with sophisticated mathematical models and efficient algorithms (Cooper and Coronella 2005, Kia and Aminian 2017). The faster and more accurate CFD simulations of fluidization systems, makes it easier to get detailed predictions compared to the expensive and time consuming experiments. On the other hand, CFD is a smart tool in optimizing the geometry, which is difficult or even impossible to achieve with experiments. Further, it provides an insight into the bed interior, which again is difficult to achieve with experiments unless more advanced technologies are used. Extreme operational conditions can also be analyzed in advanced to guarantee the safe operation of experimental setups.

However, modeling of gas-solid flow behavior is challenging due to the complexities arising from the coupling of turbulent gas flow and particle motions together with inter-particle collisions. The differences in scale from particles to geometry is another difficult parameter in the CDF simulations. Lagrangian-Eulerian and Eulerian-Eulerian are the basic modeling approaches in gas-solid multiphase systems. Lagrangian-Eulerian modeling solves the Newtonian equation of motion for each individual particle in the system while the gas phase is modeled as a continuum with Navier-Stokes equations. In contrast, the EulerianEulerian modeling considers both phases as continuous and interpenetrating, which are modeled with the
Navier-Stokes equations (Xie, Zhong et al. 2013). Even though Eulerian-Eulerian modeling consumes less computer power, it is complex in modeling stage, as it needs more closure functions. In contrast, the Lagrangian-Eulerian simulations need high computer power, and it is unrealistic to use for industrial scale reactors. Multiphase particle-in-cell (MP-PIC) was developed as an extension to the Lagrangian-Eulerian simulations, where particle are modeled in both discrete and continuous phase (Snider 2001, Xie, Zhong et al. 2013). Instead of individual particles, it considers groups of particles sharing common characteristics. These groups are referred to as parcels or computational particles. Particle properties that are best calculated on the grid are calculated using continuous modeling in the advanced time step and interpolated back to individual particles. The successive development of the MP-PIC method is illustrated in the works of Snider, O'Rourke and Andrew s (Andrews and O'Rourke 1996, Snider, O'Rourke et al. 1998, Snider 2001, Snider 2007, O'Rourke and Snider 2012). This particular method is embedded in Barracuda VR commercial software package, which is becoming popular in CFD modeling of gas-solid systems and has brought forward the concept of computational particle fluid dynamics (CPFD). Hence, the objective of this work is to analyze the effect of particle properties of density, size and size distribution on the minimum fluidization velocity with CPFD simulation.

\section{Methods and Computational Setup}

Barracuda VR 17.1.0 was used for the simulations where a simple cylindrical geometry of $1000 \mathrm{~mm}$ in height and $84 \mathrm{~mm}$ in diameter was considered. A uniform grid was applied with 8000 cells in total, which is illustrated in Figure 1. Grid refinements at the wall was not performed as it was assumed that there was no boundary layer formation with the dense phase particle system. Default grid generator settings were used, which removes the cells having less fraction of volume than 0.04 and greater aspect ratio than 15:1.

Isothermal temperature of $300 \mathrm{~K}$ was used where sand $\left(\mathrm{SiO}_{2}\right)$ was used as the basic bed material. However, other materials as aluminum oxide $\left(\mathrm{Al}_{2} \mathrm{O}_{3}\right)$, nickel oxide $(\mathrm{NiO})$, calcium $(\mathrm{Ca})$, ferric oxide $\left(\mathrm{Fe}_{2} \mathrm{O}_{3}\right)$, titanium oxide $\left(\mathrm{TiO}_{2}\right)$ and zirconium $\left(\mathrm{ZrO}_{2}\right)$ were used to analyze the effect of density on the minimum fluidization velocity. Air at atmospheric pressure was used as fluidizing gas in all the cases. Particles were filled up to $350 \mathrm{~mm}$ of height and the random packing option was used.

The close pack volume fraction, maximum momentum redirection from collisions, normal to wall momentum retention and tangent to wall momentum retention were set to $0.6,40 \%, 0.3$ and 0.99 respectively. Default values for the parameters in the particle stress model were kept unchanged. Blended acceleration model was activated 
for the mixtures of different particle sizes. The column was operated at atmospheric pressure where the air outlet at the top plane was defined as a pressure boundary. Inlet boundary was defined as a flow/velocity boundary with varying air velocities over time. Each velocity was maintained for 4 seconds. Further, uniform air distribution at the inlet and no particle exit from the pressure boundary were assumed. The bed pressure was monitored in the center of the bed at five different heights. The boundary conditions and the pressure monitoring points are depicted in Figure 1.

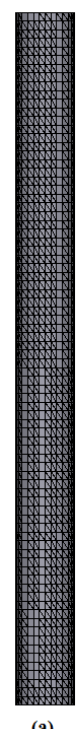

(a)
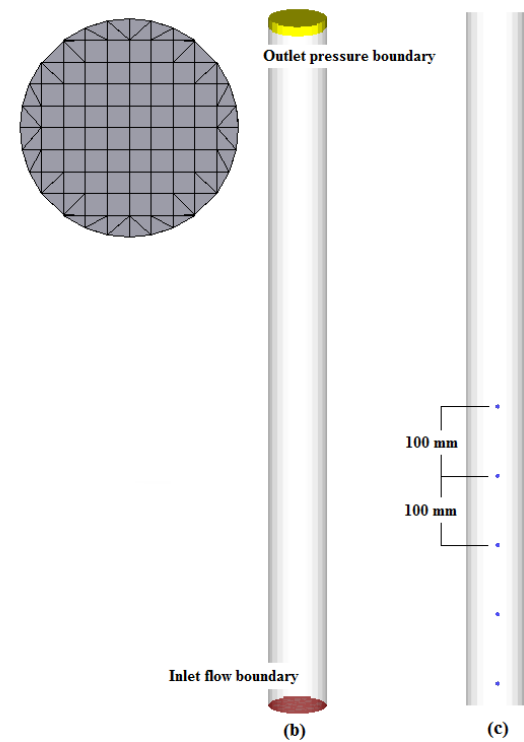

Figure 1. (a) Grid, (b) Boundary conditions, (c) Pressure data points

\section{Results and Discussion}

The bed materials used in fluidized bed gasification and combustion are usually polydispersed with a wide size distribution. However, monodispersed particle beds were used in this work to demonstrate the effect of particle size for minimum fluidization velocity. Attempts were made to analyze the effect of the particle size mixtures later in this work.

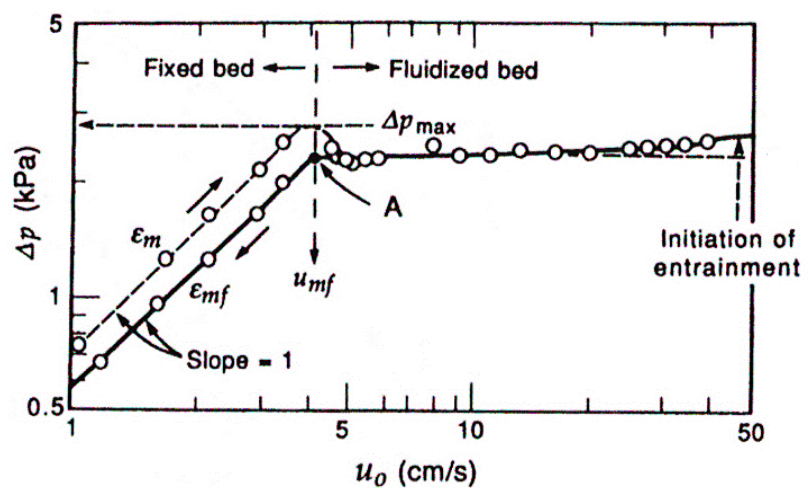

Figure 2. Gas velocity vs pressure drop diagram (Kunii and Levenspiel 1991)
The pressure drop $(\Delta \mathrm{p})$ versus superficial gas velocity $U_{0}$ diagram is useful in determining the transition from fixed bed to fluidized bed. During the fixed bed operation, the bed pressure drop is proportional to the gas velocity. Once the bed reaches the minimum fluidization velocity, the bed pressure drop decreases a little, and stabilizes at the static bed pressure. The bed continues to stay around that pressure until the particle entrainment starts (Kunii and Levenspiel 1991). This behavior and figuring out of the minimum fluidization velocity $U_{m f}$, is illustrated in Figure 2 .

Fluid drag force resulted from the upward fluid flow is one of the most important particle forces in any fluidized bed system. Due to this, many researchers have worked towards the optimization of drag models for particular cases. The author has experimentally validated the good performance of the Wen-Yu-Ergun drag model in a previous work (Bandara, Thapa et al. 2016). Gidaspow proposed a drag model where the interface momentum transfer coefficient, $K_{s g}$, is selected from either Wen-YU or Ergun correlation depending upon the gas volume fraction (Sobieski 2009). When the gas volume fraction is greater than 0.8 , Wen-Yu correlation is applied which is given by:

$$
K_{\frac{s g}{W e n_{Y u}}}=\frac{3}{4} \frac{c_{d} \rho_{g} \varepsilon_{g}\left(1-\varepsilon_{g}\right)\left(u_{s}-u_{g}\right)}{d_{p}} \varepsilon_{g}^{-2.65}
$$

Where $C_{d}$ is:

$$
C_{d}=\left\{\begin{array}{r}
\frac{24}{\varepsilon_{g} R e_{s}}\left[1+0.15\left(\varepsilon_{g} R e_{s}\right)^{0.687}\right], \quad R e_{s} \leq 1000 \\
0.44, R e_{s}>1000
\end{array}\right.
$$

When the gas volume fraction is less than 0.8, Ergun correlation is used which is given by:

$$
K_{\frac{s g}{\text { Ergun }}}=150 \frac{\mu_{g}\left(1-\varepsilon_{g}\right)^{2}}{\varphi^{2} d_{p}^{2} \varepsilon_{g}}+1.75 \frac{\rho_{g}\left(u_{g}-u_{s}\right)\left(1-\varepsilon_{g}\right)}{\varphi d_{p}}
$$

Where, subscripts $\boldsymbol{g}, \boldsymbol{p}$ and $\boldsymbol{s}$ refer to gas phase, particle and solid phase respectively. $\boldsymbol{U}$ is the velocity, $\boldsymbol{\rho}$ is the density $\boldsymbol{\varepsilon}$ is the volume fractions, $\boldsymbol{\varphi}$ is the sphericity, $\boldsymbol{\mu}$ is the viscosity, $\boldsymbol{R} \boldsymbol{e}$ is the Reynold's number and $\boldsymbol{d}$ is the particle diameter.

\subsection{Effect of the Particle Size}

Geldart has worked towards classifying the particles according to both size and density in the early 1970s (Geldart 1973). Same author has discussed the effect of the particle size distribution in fluidized beds in a separate publication. This work analyses these effects from computational fluid dynamic aspects. To demonstrate the effect of the particle size, sand particles from 400 to 800 microns were used. The particle density was $2200 \mathrm{~kg} / \mathrm{m} 3$ and it was further assumed that the particles were spherical. As shown in Figure 3, the 


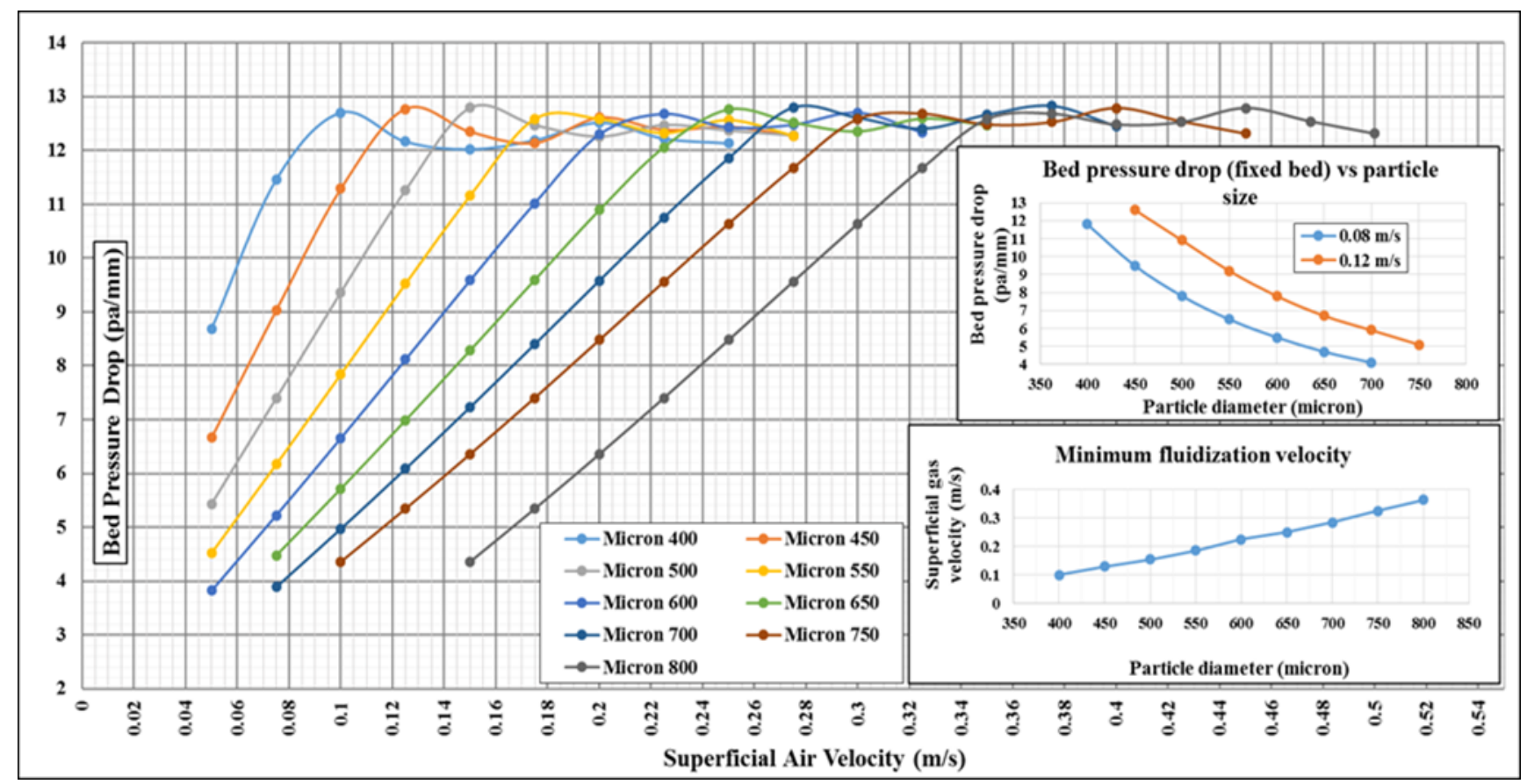

Figure 3. Minimum fluidization velocity plots for different size particles. Right upper corner sub-plot illustrates the pressure drop during fixed bed at two different gas velocities

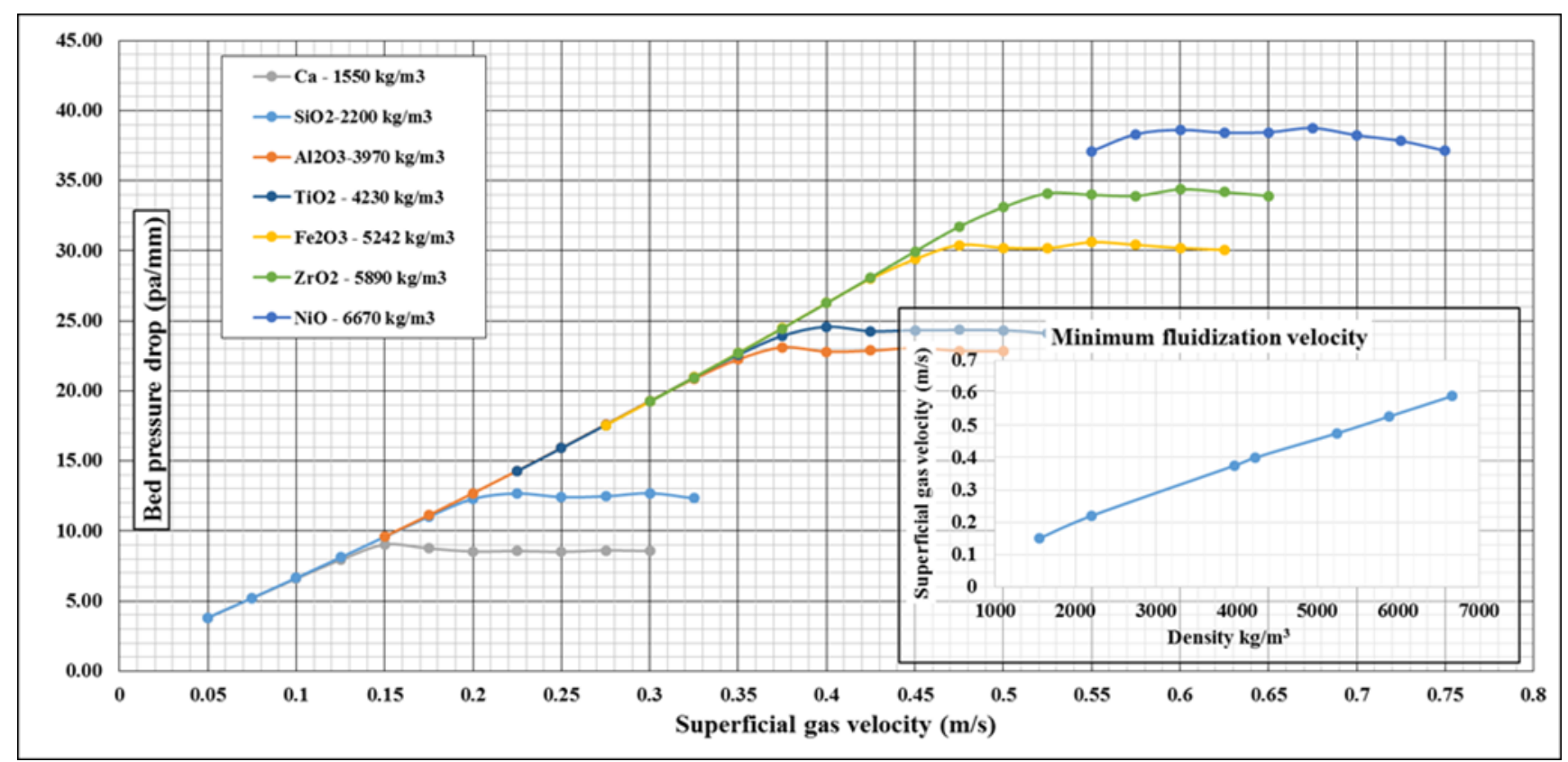

Figure 4: Effect of the particle density for minimum fluidization velocity

minimum fluidization velocity increases linearly with the particle diameter as expected. Bed pressure drop shows a linear relationship with the superficial gas velocity during the packed bed region. Further, it is clear from the figure that the bed pressure drop at the minimum fluidization is almost the same for all the particle sizes. It is also agreeable because, the bed weight is counter balanced by the pressure drop at the fluidization and the bed mass was approximately constant for all the sizes. The fluctuations of the pressure drop in the fluidizing region is also realistic which can be observed in many experimental results as well.

\subsection{Effect of the Particle Density}

A 600-micron sand bed was considered as the reference and the effect of different densities were analyzed for 600-micron particles. According to the simulation results depicted in Figure 4, the minimum fluidization velocity is proportional to the particle density. As the particle diameter is similar, all the plots follow the same line during the packed bed operation. 


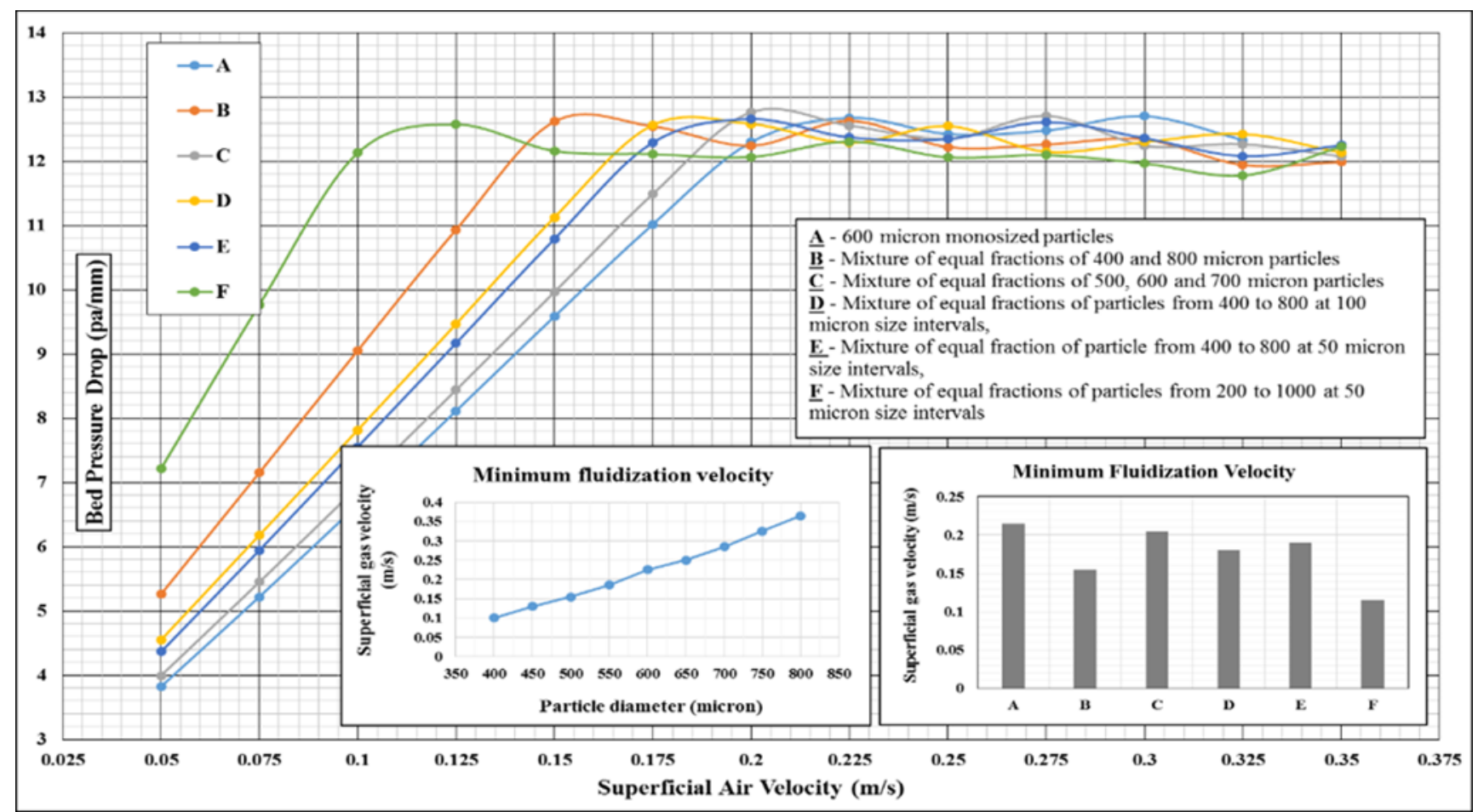

Figure 5. Effect of the particle size distribution on the minimum fluidization velocity

\subsection{Effect of the Particle Size Distribution}

The final and major task of this work is to see the functioning of CFD technique in predicting the minimum fluidization velocity behavior of particle mixtures of different sizes. Specially, empirical models for the drag force have been developed considering mono size particles. However, the particle drag force in a mixture of different size particles differs compared to that in a mono-size particle bed.

The effect of the particle size distribution was compared with the 600-micron monodispersed silica particles. Different particle mixtures of silica all with a mean particle diameter as 600 -micron were simulated. The description of the particle mixtures are given in Figure 5. Particle mixtures were defined by introducing one particle species for each size and filling them randomly with pre-defined volume fractions, which collectively accounts for 0.6 solid/particle volume fraction.

According to the same Figure 5, 600-micron monodispersed particles has the highest minimum fluidization velocity, which is approximately $0.21 \mathrm{~m} / \mathrm{s}$. The minimum fluidization velocity of mixture $\mathrm{C}$ is close to the value of the 600 -micron monodispersed sample. This might be due to the narrow size distribution of mixture $\mathrm{C}$ around 600 micron. The minimum fluidization velocity of mixtures $\mathrm{D}$ and $\mathrm{E}$ is closer to each other, but the values are less than $\mathrm{A}$ and $\mathrm{C}$. The particle sizes of $\mathrm{D}$ and $\mathrm{E}$ mixtures are distributed between 400 and 800 micron in a similar way to a certain extent. The size distribution of pre-mentioned mixtures are in a broad range with oversized and undersized particles than 600 micron. The mixture B, which is having equal fractions of 400 and 800 micron particles, shows a lower minimum fluidization velocity compared to A, C, D and E. This particular mixture contains half of the fraction with 400 microns, which is comparatively less compared to 600 . Finally, the mixture $\mathrm{F}$ with the highest size distribution (between 200 to 1000 microns) shows the lowest minimum fluidization velocity among the six different mixtures considered.

It is important to note the possibility of reducing the minimum fluidization velocity by adding a certain fraction of smaller sized particles. As an example, even though the mixture F contains considerable amounts of particles larger than 600 micron, the minimum fluidization velocity still substantially drops below the value of monodispersed 600 microns sample. In this situation, the larger particles are affected both by the fluid drag force and by the momentum from smaller particles (particle drag). According to the simulations carried out for different diameters, smaller particles are prone to fluidized at lower gas velocities. Therefore, the drag force from the fine particles make the larger particles fluidize at lower gas velocities when those are in a mixture. However, the simulation time was increased considerably for particle mixtures than monodispersed particle beds.

\section{Conclusion}

The effects of particle size, density and size distribution on the minimum fluidization velocity were analyzed using the MP-PIC CFD simulation technique. Barracuda VR commercial software package was used in all the simulations. A previously validated model, which uses 
the Wen-YU-Ergun model for the fluid drag, was used. However, the model had not been validated for particles with size distribution for several sizes. It is good to conduct experimental analysis further to guarantee the reproducibility of simulation data.

Minimum fluidization velocity was observed to be linearly proportional to the particle size. On the other hand, the minimum fluidization velocity increases approximately by a factor of two when the particle density is doubled. It is the pressure drop, which is more concerned during packed bed operation. Simulation results for different size and densities prove that it is the particle size, which governs the pressure drop. However, it is not easy to observe a clear relationship between minimum fluidization velocity and particle sizes when it comes to particle mixtures. The smaller particles in a mixture greatly affects and reduces the minimum fluidization velocity. Thus, this phenomenon is useful in operating a bubbling fluidized bed reactor at different gas velocities, simply by adding either larger or smaller particles depending on the requirement. Finally, the Barracuda CPFD simulations can provide precise and quick insight into the bed hydrodynamics.

\section{Acknowledgement}

The authors would like to forward their gratitude to University College of Southeast Norway for providing of the Barracuda VR software package and computer facilities.

\section{References}

Andrews, M. J. and P. J. O'Rourke (1996). "The multiphase particle-in-cell (MP-PIC) method for dense particulate flows." International Journal of Multiphase Flow 22(2): 379-402.

Bandara, J. C., R. K. Thapa, B. M. E. Moldestad and M. S. Eikeland (2016). "Simulation of Particle Segregation in Fluidized Beds" 9th EUROSIM Congress on Modelling and Simulation, Oulu, Finland, IEEE.

Behjat, Y., S. Shahhosseini and S. H. Hashemabadi (2008). "CFD modeling of hydrodynamic and heat transfer in fluidized bed reactors." International Communications in Heat and Mass Transfer 35(3): 357-368.

Coltters, R. and A. L. Rivas (2004). "Minimum fluidation velocity correlations in particulate systems." Powder Technology 147(1-3): 34-48.

Cooper, S. and C. J. Coronella (2005). "CFD simulations of particle mixing in a binary fluidized bed." Powder Technology 151(1-3): 27-36.

Cui, H. and J. R. Grace (2007). "Fluidization of biomass particles: A review of experimental multiphase flow aspects." Chemical Engineering Science 62(1-2): 45-55.

Esmaili, E. and N. Mahinpey (2011). "Adjustment of drag coefficient correlations in three dimensional CFD simulation of gas-solid bubbling fluidized bed." Advances in Engineering Software 42(6): 375-386.

Fotovat, F., R. Ansart, M. Hemati, O. Simonin and J. Chaouki (2015). "Sand-assisted fluidization of large cylindrical and spherical biomass particles: Experiments and simulation." Chemical Engineering Science 126: 543-559.

Geldart, D. (1972). "The effect of particle size and size distribution on the behaviour of gas-fluidised beds." Powder Technology 6(4): 201-215.

Geldart, D. (1973). "Types of gas fluidization." Powder Technology 7(5): 285-292.

Horio, M. (2013). 1 - Overview of fluidization science and fluidized bed technologies A2 - Scala, Fabrizio. Fluidized Bed Technologies for Near-Zero Emission Combustion and Gasification, Woodhead Publishing: 3-41.

Kia, S. A. and J. Aminian (2017). "Hydrodynamic modeling strategy for dense to dilute gas-solid fluidized beds." Particuology 31: 105-116.

Kunii, D. and O. Levenspiel (1991). Fluidization Engineering. Washington Street, Newton, MA, Butterworth-Heinemann.

O'Rourke, P. J. and D. M. Snider (2012). "Inclusion of collisional return-to-isotropy in the MP-PIC method." Chemical Engineering Science 80: 39-54.

REN21 (2016). Renewables 2016 Global Status Report.

Snider, D. M. (2001). "An Incompressible Three-Dimensional Multiphase Particle-in-Cell Model for Dense Particle Flows." Journal of Computational Physics 170(2): 523-549.

Snider, D. M. (2007). "Three fundamental granular flow experiments and CPFD predictions." Powder Technology 176(1): 36-46.

Snider, D. M., P. J. O'Rourke and M. J. Andrews (1998). "Sediment flow in inclined vessels calculated using a multiphase particle-in-cell model for dense particle flows." International Journal of Multiphase Flow 24(8): 13591382.

Sobieski, W. (2009). "Switch Function and Sphericity Coefficient in the Gidaspow Drag Model for Modeling Solid-Fluid Systems." Drying Technology 27(2): 267-280.

Vollmari, K., R. Jasevičius and H. Kruggel-Emden (2016). "Experimental and numerical study of fluidization and pressure drop of spherical and non-spherical particles in a model scale fluidized bed." Powder Technology 291: 506521.

Winter, F. and B. Schratzer (2013). 23 - Applications of fluidized bed technology in processes other than combustion and gasification A2 - Scala, Fabrizio. Fluidized Bed Technologies for Near-Zero Emission Combustion and Gasification, Woodhead Publishing: 1005-1033.

Xie, J., W. Zhong, B. Jin, Y. Shao and Y. Huang (2013). "Eulerian-Lagrangian method for three-dimensional simulation of fluidized bed coal gasification." Advanced Powder Technology 24(1): 382-392. 